\title{
Escalas representadas em gráficos: Um estudo de intervenção com alunos do $5^{\circ}$ ano
}

\author{
Maria Betânia Evangelistai \& Gilda Lisbôa Guimarãesii \\ Universidade Federal de Pernambuco, Brasil
}

\begin{abstract}
Resumo
Este artigo apresenta uma pesquisa que investigou a aprendizagem sobre escala representada em gráficos de barras e linhas a partir de uma intervenção de ensino. Participaram no estudo 69 alunos do $5 .^{\circ}$ ano (aproximadamente, 10 anos de idade) de três escolas públicas brasileiras. Para cada grupo, foram propostas atividades envolvendo diferentes situações: medida de comprimento, reta numérica e mapas. Todos os alunos participaram num pré-teste, uma intervenção de ensino e um pós-teste. Os resultados revelaram um desconhecimento dos alunos, que demonstraram dificuldades em representar, localizar, analisar, comparar e construir escalas em gráficos. Porém, após apenas duas sessões de intervenção em cada turma, foram observados avanços significativos na aprendizagem de todos os grupos. Assim, podemos afirmar que alunos dos anos iniciais, quando levados a refletir sobre escalas, são capazes de aprender sobre essa representação. Dessa forma, evidencia-se a necessidade e a possibilidade de um trabalho sistemático nas escolas.
\end{abstract}

Palavras-chave

Escala; Gráfico; Ensino Fundamental; Matemática

\section{Introdução}

A crescente necessidade de levar os alunos a se apropriarem de conceitos estatísticos se justifica pela atual possibilidade de se analisar uma 
grande quantidade de dados, em função do advento de computadores e software disponíveis para tal. Além disso, o ensino de Estatística teve sua inclusão realizada recentemente nos currículos brasileiros para os anos iniciais de escolarização. Acrescido a isso, ressaltamos que quando o aluno realiza pesquisas, ele pode refletir autonomamente e, consequentemente, ser capaz de interpretar a realidade a partir de análises de dados coletados ou de interpretar de forma crítica dados sistematizados em tabelas e gráficos.

Ponte, Brocardo, e Oliveira (2009) argumentam que a Estatística exerce um papel essencial na educação para a cidadania. Essa pode ser considerada uma importante ferramenta para a realização de projetos e investigações em diversos campos, sendo usada no planejamento, na coleta e análise de dados, nas realizações de inferências para se tomar decisões com o intuito de apoiar afirmações em diversas áreas, como saúde, educação, ciência e política.

Entretanto, como é evidenciado pelo Indicador de Alfabetismo Funcional - INAF (D’Ambrósio, 2007), apenas $27 \%$ da população brasileira é capaz de interpretar informações apresentadas em gráficos e, destes, 62\% chegaram a cursar pelo menos o Ensino Médio. Assim, fica explícito que a compreensão de gráficos não tem sido um conhecimento que as experiências de vida têm ajudado a construir. Cabe à escola esse papel essencial do processo de apropriação dos conteúdos estatísticos.

O Grupo de Estudo em Educação Estatística no Ensino Fundamental - GREF, da Universidade Federal de Pernambuco, vem desenvolvendo várias pesquisas no intuito de contribuir com a aprendizagem dos alunos sobre estatística no Ensino Fundamental. Este artigo apresenta um desses estudos, o qual buscou investigar, especificamente, a possibilidade de aprendizagem sobre escala representada em gráficos de barras e linhas, por alunos que cursavam o $5 .^{\circ}$ ano do Ensino Fundamental.

\section{O conceito de escala}

O conceito de escala pode ser utilizado em diferentes áreas de conhecimento, como Geografia, Matemática, Cartografia, Engenharia, entre outras. De forma intencional ou não, lidamos constantemente com a noção de escala em nosso dia a dia, mediante a leitura de mapas, de gráficos, de plantas de imóvel, na utilização de instrumentos de medições e outros. 
Em Matemática, o conceito de escala é trabalhado quando se abordam números racionais, proporcionalidade, semelhança de figuras, leitura de gráficos, comprimento, área, estruturas multiplicativas, construção de figuras, como foi verificado por Melo e Bellemain (2004). Os Parâmetros Curriculares Nacionais de Matemática para o $1 .^{\circ}$ e $2 .^{\circ}$ ciclos do Ensino Fundamental (MEC/SEF, 1997) fazem referência ao conceito de escala em todos os eixos temáticos: números e operações; espaço e forma; grandezas e medidas; e tratamento da informação.

Entretanto, o conceito de escala nem sempre é explorado de forma intencional e sistemática na escola, como argumentam Melo e Bellemain (2006). Isso pode ser um fator determinante para se justificar a dificuldade dos alunos em atividades que requerem as habilidades de representar, de localizar, de analisar, de comparar e de construir escalas com diferentes intervalos representadas em gráficos de barras e linhas.

Melo e Bellemain (2009) definem escala gráfica como sendo uma representação de várias distâncias sobre uma linha reta graduada, uma vez que é representada por um segmento reto dividido em submúltiplos da unidade escolhida, graduada da esquerda para a direta.

A aprendizagem sobre escalas representadas em gráficos tem sido considerada uma das maiores dificuldades das pessoas ao interpretarem ou construírem gráficos, como afirma Ainley (2000). Várias pesquisas apontam que tanto alunos como professores dos anos iniciais apresentam dificuldades com relação à interpretação e construção de gráficos (Guimarães, 2002; Lemos, 2002; Albuquerque, 2010).

Estudos como os de Guimarães, Ferreira, e Roazzi (2001), Albuquerque (2010), Lima (2010) e Silva (2012) buscaram verificar a compreensão de alunos de diferentes níveis de escolaridade sobre escala representada em gráficos. Esses concluíram que a dificuldade dos estudantes com valores implícitos e com a proporcionalidade da escala era bastante evidente. Conforme Albuquerque (2010) e Silva (2012), alunos dos anos iniciais de escolarização, sejam adultos ou crianças, sentem bastante dificuldade para realizar atividades que requerem as habilidades de interpretação e de construção de escalas em gráficos, principalmente quando os valores registrados na escala não estão explícitos. Guimarães (2002) e Albuquerque (2010) ressaltam que os alunos não compreendem que existe uma continuidade numérica entre os intervalos da escala, apresentando muita 
dificuldade para estabelecer a proporcionalidade entre os valores da escala. Assim, destacam a necessidade de um trabalho sistemático e interrelacionado, que leve o aluno a refletir sobre a importância e a funcionalidade da utilização da escala, bem como a compreender a grandeza comprimento, discutindo as unidades de medida e suas subunidades.

Além disso, é fundamental ressaltar que escalas representadas nos gráficos podem fornecer imagens distorcidas sobre uma determinada informação. Cavalcanti, Natrielli, e Guimarães (2010), ao analisarem os gráficos veiculados na mídia impressa, considerando três tipos de suportes, constataram que $39 \%$ dos gráficos analisados apresentavam escalas com proporcionalidades inadequadas, as quais poderiam levar os leitores a compreensões equivocadas da real informação. Como argumenta Monteiro (2006), os gráficos vêm sendo utilizados frequentemente pelos meios de comunicação de massa e, de acordo com a intencionalidade de quem apresenta as informações, podem encobrir ou realçar determinados aspectos da notícia. Desse modo, compreender o conceito de escala é fundamental, para que os alunos possam avaliar de forma crítica as informações mostradas nesse tipo de representação.

Neste contexto, Boaventura e Fernandes (2004) e Ponte, Brocardo, e Oliveira (2009) vêm defendendo que a Estatística deve ser vista como linguagem de descrição e interpretação da realidade, bem como merece uma atenção no âmbito escolar. Diante disso, percebemos a necessidade de realizar um estudo de intervenção, com intuito de investigar a possibilidade de aprendizagem sobre escala representada em gráficos de barras e de linha por alunos dos anos iniciais. E, para tal, era necessário definir que sequência de atividades poderia ser proposta para a aprendizagem.

Para a construção das atividades trabalhadas tomamos como base o levantamento realizado por Evangelista e Guimarães (2013). Nesse estudo, as autoras investigaram quais eram as atividades que apresentavam o conceito de escala em 5 (cinco) coleções de livros didáticos de Matemática, recomendados pelo PNLD $^{1} 2013$, nos volumes do $4 .^{\circ}$ e $5 .^{\circ}$ anos do Ensino Fundamental. As autoras perceberam que o conceito de escala estava presente nos quatros eixos temáticos de matemática do currículo brasileiro (Números e Operações; Espaço e Forma; Grandezas e Medidas; e Tratamento da Informação), possibilitando uma riqueza de conexões entre esses conteúdos. 
A partir desse levantamento, optámos por uma intervenção que envolvesse atividades propostas nos livros didáticos, considerando esses diferentes eixos (medidas de comprimento/eixo grandezas e medidas; reta numérica/eixo números e operações e mapas/eixo de espaço e forma), buscando investigar se essas atividades contribuiriam para a aprendizagem sobre escalas representadas em gráficos.

\section{Método}

Esta pesquisa foi realizada em três turmas (MC, RN e MP) de diferentes escolas públicas municipais da Região Metropolitana de Recife Brasil. Nessas escolas estudam alunos de baixa renda que moram nas redondezas. Participaram ao todo 69 alunos do $5 .^{\circ}$ ano do Ensino Fundamental (crianças com aproximadamente 10 anos de idade), ou seja, alunos que estavam finalizando os anos iniciais de escolarização. Nas turmas dos anos iniciais existe apenas um professor, que ministra conteúdos de diferentes disciplinas (português, matemática, ciências, história, geografia e outras).

Assim, iniciámos avaliando o nível de conhecimento dos alunos sobre escala, solicitando que respondessem, individualmente, a um pré-teste envolvendo atividades de interpretação e construção de escalas representadas em gráficos de barras e de linhas. As questões são apresentadas mais adiante.

Em cada turma foi realizada, por uma das pesquisadoras, uma intervenção de ensino compreendida de duas sessões. Cada turma teve um contexto de intervenção: medidas de comprimento $(\mathrm{MC})$; reta numérica $(\mathrm{RN})^{2}$; e mapas (MP). Esses contextos estão presentes nas atividades dos livros didáticos brasileiros, de acordo com Evangelista e Guimarães (2013), como mencionado anteriormente. Dessa forma, para esse estudo optámos por realizar a intervenção de ensino considerando os três tipos de situações que não envolviam diretamente a representação em gráficos, para justamente investigar a possibilidade dessas atividades, usualmente propostas nos livros didáticos, contribuírem para a aprendizagem de leitura e interpretação de escalas representadas em gráficos de barras e de linhas e se cada uma delas auxiliava de uma forma específica. 
Cada sessão teve a duração de aproximadamente duas horas. Uma das pesquisadoras, professora efetiva dos anos iniciais de outra escola pública, realizou a intervenção em cada uma das turmas, a partir de atividades resolvidas em duplas e posterior reflexão com a turma toda. No primeiro dia, foram trabalhadas atividades envolvendo interpretação de valores na escala e, no segundo dia, foram exploradas atividades de construção de escalas. Para garantir a compreensão dos enunciados pelos alunos que ainda apresentavam dificuldades com leitura, a pesquisadora/professora realizava a leitura das questões. Após a execução de cada atividade, era realizada uma correção coletiva no quadro, a qual buscava estimular uma reflexão, por parte dos alunos, acerca dos valores explícitos apresentados na escala, suas subdivisões e quais poderiam ser os valores intermediários, tendo prioritariamente como referência a metade, metade da metade e intervalos múltiplos de 10 e 5.

Salientamos que os valores representados nos questionamentos variavam de acordo com as situações exploradas em cada atividade e com o intervalo representado nas escalas utilizadas. Seguem exemplos das atividades exploradas na intervenção de cada grupo. As atividades foram selecionadas e adaptadas de livros didáticos de Matemática, recomendados pelo Programa Nacional do Livro Didático 2013 , nos volumes do $4 .^{\circ}$ e $5 .^{\circ}$ anos do Ensino Fundamental.

$\mathrm{Na}$ Figura 1 apresentamos o exemplo de uma atividade que foi trabalhada com os alunos no contexto de medida de comprimento.

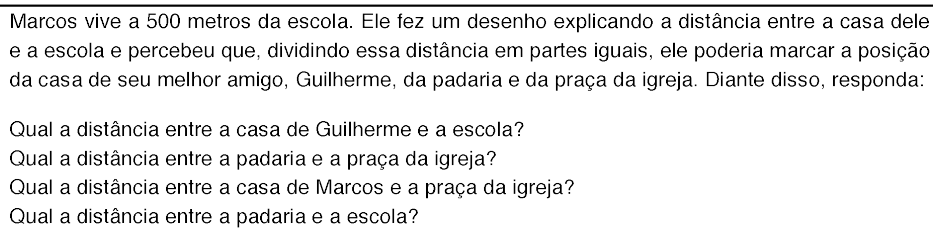

Figura 1 - Atividade de interpretar escala no contexto de medida de comprimento 
$\mathrm{Na}$ Figura 2 apresentamos o exemplo de uma atividade que foi trabalhada com os alunos no contexto de reta numérica.

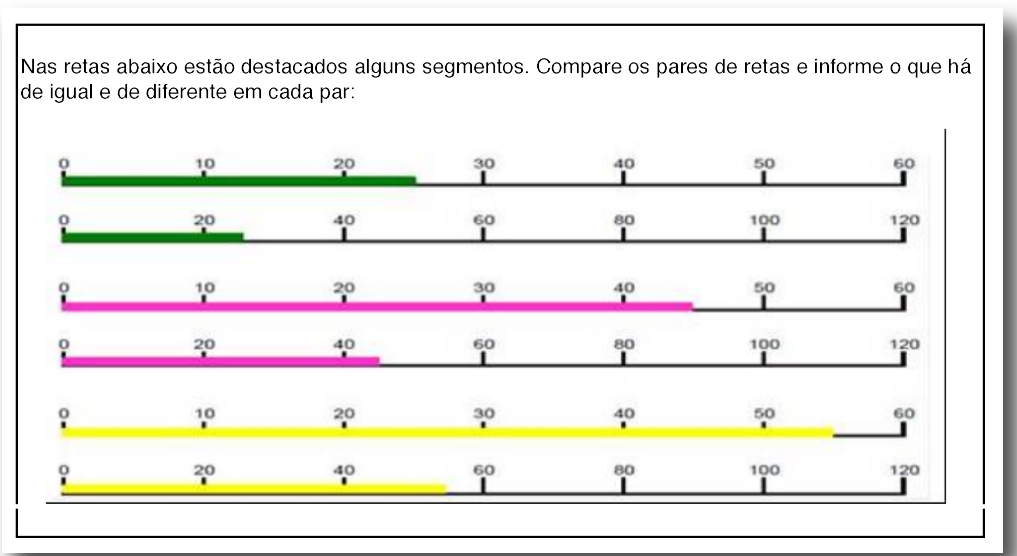

Figura 2 - Atividade de interpretar escala no contexto de reta numérica

Na Figura 3 mostramos o exemplo de uma atividade que foi trabalhada com os alunos no contexto de mapas.

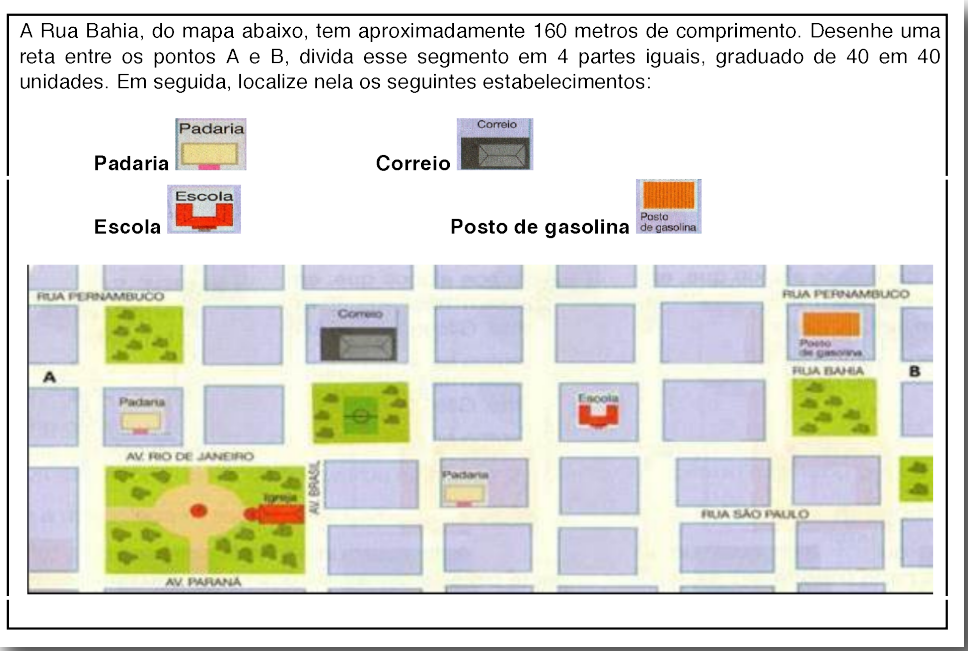

Figura 3 - Atividade de construção de escala no contexto de mapa 
No final da intervenção foi realizado um pós-teste, para avaliar possíveis avanços na aprendizagem dos alunos. As questões são apresentadas mais adiante.

Todas as atividades trabalhadas nos testes (pré e pós) e nas intervenções foram selecionadas e/ou adaptadas de atividades exploradas em livros didáticos do $4 .^{\circ}$ e do $5 .^{\circ}$ anos de escolaridade. As atividades envolviam: representação em gráfico de barras e linha; localização de valores implícitos na escala; identificação de erros cometidos intencionalmente em gráfico de barras; e construção de gráfico a partir de uma tabela. As informações eram também apresentadas com intervalos escalares diferentes.

\section{Resultados}

Como pode ser observado no Gráfico 1, os três grupos apresentaram um desempenho muito baixo no pré-teste, uma vez que a pontuação máxima poderia ser de 9 (nove) pontos. Os desempenhos dos grupos não apresentaram diferenças significativas $[F(2,68)=1,285 ; p=0,283]$. Esses dados ratificam estudos anteriores, os quais afirmam a dificuldade de alunos dos anos iniciais de escolarização em compreender uma escala (Guimarães, 2002; Albuquerque, 2010; Lima, 2010; Silva, 2012).

Entretanto, após a realização da intervenção de ensino, todos os grupos apresentaram avanços significativos entre o pré e pós-teste: [t (23) = 4,$143 ; p \leq 0,000$ ] para o grupo $\mathrm{MC}$, [t $(22)=-7,497 ; \mathrm{p} \leq 0,000$ ] para o grupo $\mathrm{RN}$ e $[\mathrm{t}(21)=-3,813 ; \mathrm{p}<0,001]$ para o grupo MP.

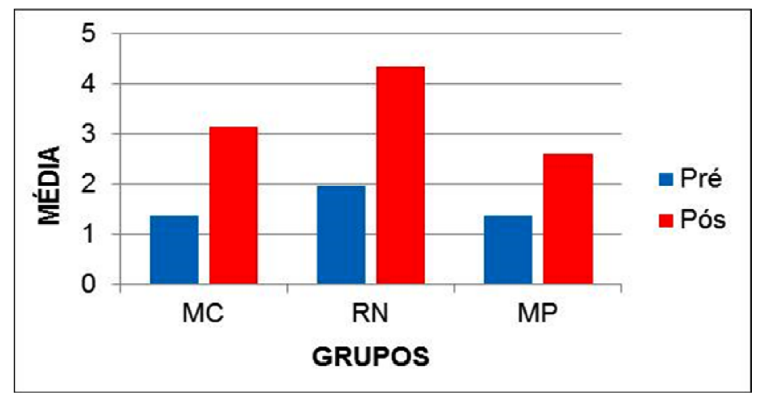

Gráfico 1 - Média de acertos nos testes por grupo e por fase 
Dessa forma, a intervenção realizada em cada um dos grupos contribuiu efetivamente para a aprendizagem sobre escalas representadas em gráficos, independentemente do tipo de contexto apresentado na intervenção de ensino. Tais resultados nos parecem muito importantes, uma vez que expressam a facilidade que as crianças apresentam em aprender sobre escalas quando são estimuladas de forma sistemática. Assim, com apenas duas sessões de intervenção, duas aulas de aproximadamente 2 horas, todas as turmas apresentaram progresso significativo.

Além disso, podemos afirmar que os três tipos de situação por nós propostos (medida de comprimento, reta numérica e mapas), os quais pertencem a diferentes eixos da matemática, permitiram a aprendizagem sobre escalas e a compreensão dessas representadas em gráficos de linha e barra. Esse resultado evidencia a possibilidade da aprendizagem a partir do recurso que elas já possuem, ou seja, o livro didático. O que precisa ser valorizado é uma reflexão sistemática sobre escala.

Lima e Magina (2010) também conseguiram avanço positivo ao trabalharem com alunos do $5 .^{\circ}$ ano, realizando uma intervenção de ensino sobre representação gráfica no ambiente informatizado Tabletop. Como em nosso estudo, as autoras afirmam que a intervenção de ensino favoreceu a compreensão dos alunos com relação à localização de pontos implícitos na escala.

Dessa forma, observa-se que quando os alunos são levados a refletir sobre escala, sobre os valores explícitos e implícitos da gradação da mesma e sobre a importância da proporcionalidade dos intervalos, é possível promover a aprendizagem sobre escala.

Entretanto, apesar da aprendizagem significativa dos alunos de todos os grupos, verifica-se que eles precisam avançar ainda mais. Acreditamos que uma intervenção sistemática mais prolongada ou um trabalho desenvolvido desde os primeiros anos de escolaridade sobre a aprendizagem de conceitos estatísticos, entre eles o de escala, poderá levar os alunos a uma maior compreensão.

Uma vez analisado o desempenho geral dos grupos, passamos a analisar o desempenho em cada uma das questões. Essa análise permite evidenciar que habilidades foram mais facilmente compreendidas. 
Na primeira questão foi solicitado aos alunos que representassem três valores na escala de um gráfico de barra (Figura 4). No pré-teste os percentuais de acertos obtidos pelos grupos foram baixos (MC 12,5\%; RN 34,8\%; MP 13,6\%). Após a intervenção de ensino, todos os grupos melhoraram seus desempenhos (MC 45,8\%; RN 56,5\%; MP 31,8\%), especialmente o grupo $\mathrm{MC}$, que apresentou uma diferença significativa $(\mathrm{p}=$ $0,008, n=24$ ) entre o pré-teste e o pós-teste, conforme o teste McNemar.

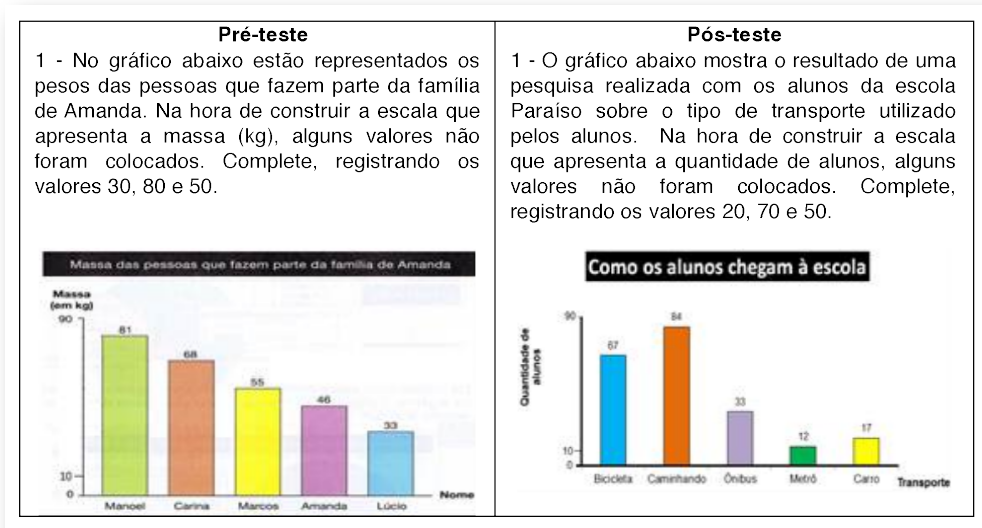

Figura 4 - Primeira questão do pré-teste e do pós-teste

$\mathrm{Na}$ segunda questão foi solicitado novamente que os alunos representassem três valores na escala, agora num gráfico de linha simples (Figura 5). Novamente, no pré-teste todos os grupos apresentaram um baixo desempenho (MC 12,5\%; RN 21,7\%; MP 13,6\%). Já no pós-teste constatamos avanços em todos os grupos (MC 41,7\%; RN 65,2\%; MP 31,8\%), principalmente nos grupos $\mathrm{MC}$ e RN, que apresentaram diferença significativa entre as fases $(p=0,039, n=24)$ e $(p=0,002, n=23)$, respectivamente. 


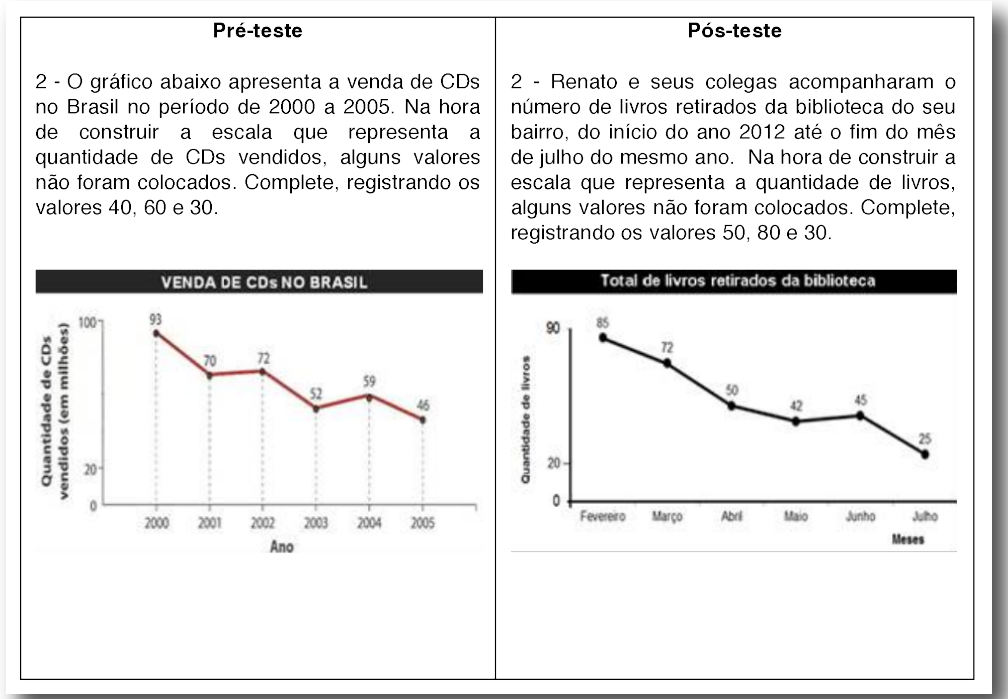

Figura 5 - Segunda questão do pré-teste e do pós-teste

Como na primeira e na segunda questões trabalhamos a mesma habilidade, diferenciando apenas o tipo de gráfico (barras e linha), é importante realizarmos uma comparação entre as mesmas. Segundo Albuquerque (2010), os alunos têm mais facilidade em interpretar informações representadas em gráficos de barras do que em gráficos de linha. Essa situação pode ser explicada pela pouca frequência de atividades nos livros didáticos de Matemática que explorem os gráficos de linhas, como foi levantado por Guimarães, Gitirana, Cavalcanti, e Marques (2007). Acrescido a isso, os gráficos de barras também são os mais explorados pela mídia impressa, o que os torna mais familiares para os alunos do que os de linhas.

Entretanto, observamos que os percentuais de acertos nas duas questões são semelhantes e não apresentam diferenças significativas. Assim, contrariando os estudos anteriores, constatamos que, independente do tipo de gráfico (barras ou linha) utilizado nas atividades do pré-teste e pós-teste, que exploravam representações de valores na escala, os grupos apresentaram desempenhos semelhantes, ou seja, o tipo de gráfico não foi um fator que determinou o desempenho dos grupos. 
Guimarães (2002), trabalhando com alunos do $4 .^{\circ}$ ano, Albuquerque (2010), com alunos do $5 .^{\circ}$ ano e Lima e Magina (2010), também com alunos do $5 .^{\circ}$ ano do Ensino Fundamental, afirmaram que localizar valores implícitos na escala é uma habilidade em que os alunos sentem bastante dificuldade. Essas autoras argumentam que essa dificuldade pode estar relacionada à compreensão dos valores contínuos apresentados numa reta numérica e à necessidade de se estabelecer a proporcionalidade entre os pontos.

Assim, na terceira questão, foi solicitado aos alunos que localizassem valores implícitos na escala de um gráfico de barras (Figura 6). No pré-teste todos os grupos apresentaram um fraco desempenho (MC 8,3\%; RN 8,7\%; MP 9,1\%). Entretanto, após a intervenção de ensino, constatamos um grande progresso no desempenho de todos os grupos (MC 58,3\%; RN 52,2\%; MP $27,3 \%$ ), principalmente os grupos $M C$ e $R N$, que apresentaram diferenças significativas: $M C(p \leq 0,000, n=24)$ e $R N(p=0,006, n=23)$.

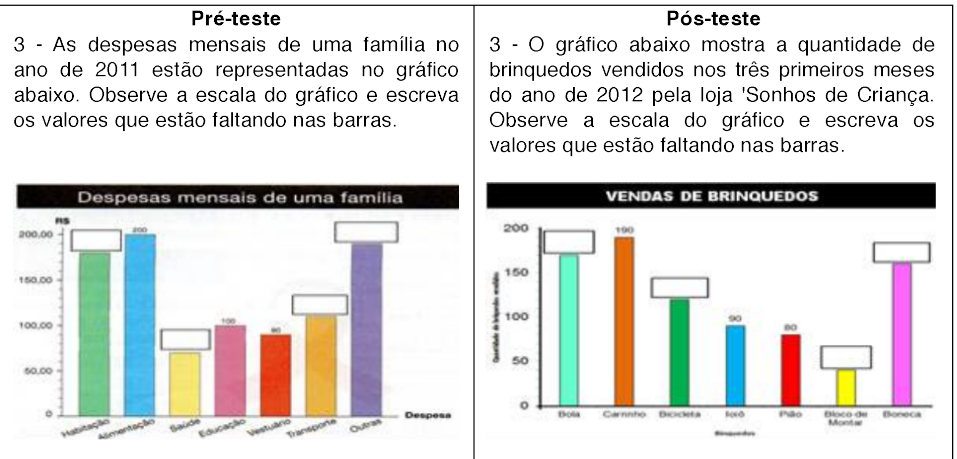

Figura 6 - Terceira questão do pré-teste e do pós-teste

Esses resultados indicam que as intervenções de ensino às quais os alunos foram submetidos contribuíram consideravelmente para que os mesmos localizassem os valores implícitos na escala do gráfico de barras.

Bruno e Espinel (2005) observaram que, para os alunos, interpretar pontos na reta numérica era uma atividade mais fácil do que representá-los em uma escala sem graduação, evidenciando que estabelecer a 
proporcionalidade entre os valores de uma reta numérica é uma das maiores dificuldades.

Dessa forma, comparamos o desempenho de nossos alunos em representar ou localizar valores na escala em um gráfico de barras (primeira e terceira questão). Observamos que, no pós-teste, os alunos apresentaram um avanço no desempenho de ambas as questões, independentemente das habilidades exigidas, o que mostra que os alunos foram igualmente capazes de aprender a representar e localizar valores na escala.

$\mathrm{Na}$ quarta questão foi solicitado aos alunos que localizassem valores implícitos na escala de um gráfico de linha simples (Figura 7). Observamos que todos os grupos apresentaram um fraco desempenho no pré-teste (MC 12,5\%; RN 17,4\%; MP 9,1\%). Entretanto, após a intervenção, todos os grupos conseguiram avançar (MC 29,2\%; RN 43,5\%; MP 13,6\%), evidenciando que o trabalho realizado nas sessões de intervenção contribuiu para melhorar o desempenho dos alunos em relação à localização de valores implícitos na escala também em gráfico de linha, principalmente o grupo $\mathrm{RN}$, que apresentou uma diferença significativa entre as fases $(p=0,031, n=23)$.

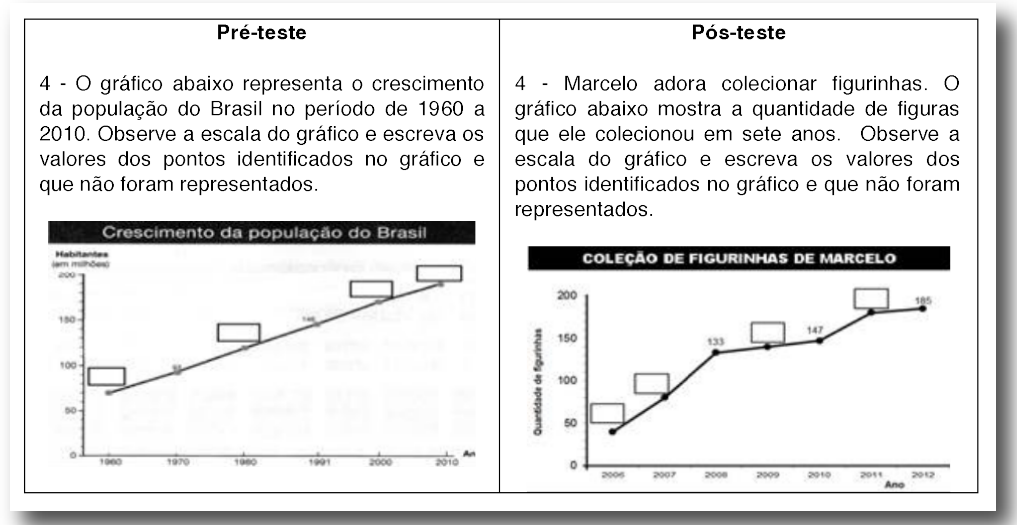

Figura 7 - Quarta questão do pré-teste e do pós-teste

Porém, ao compararmos o desempenho na localização de valores implícitos na escala em gráfico de barra e em gráfico de linha (terceira e quarta questões), observamos que todos os grupos apresentaram um melhor 
desempenho ao localizar valores no gráfico de barras. Assim, apesar das atividades exploradas na intervenção terem auxiliado tanto na representação quanto na localização de valores implícitos na escala de gráficos de barra e de linha simples, localizar dados foi mais fácil no gráfico de barras do que no de linhas.

Na quinta questão (Figura 8) foi solicitado aos alunos que localizassem erros em uma escala, os quais foram cometidos intencionalmente. Observamos que antes da realização da intervenção os percentuais de acerto eram baixos (MC 8,3\%; RN 26,1\%; MP 27,3\%) e após a realização da intervenção todos os grupos avançaram significativamente (MC 20,8\%; RN $52,2 \%$; MP 54,5\%).

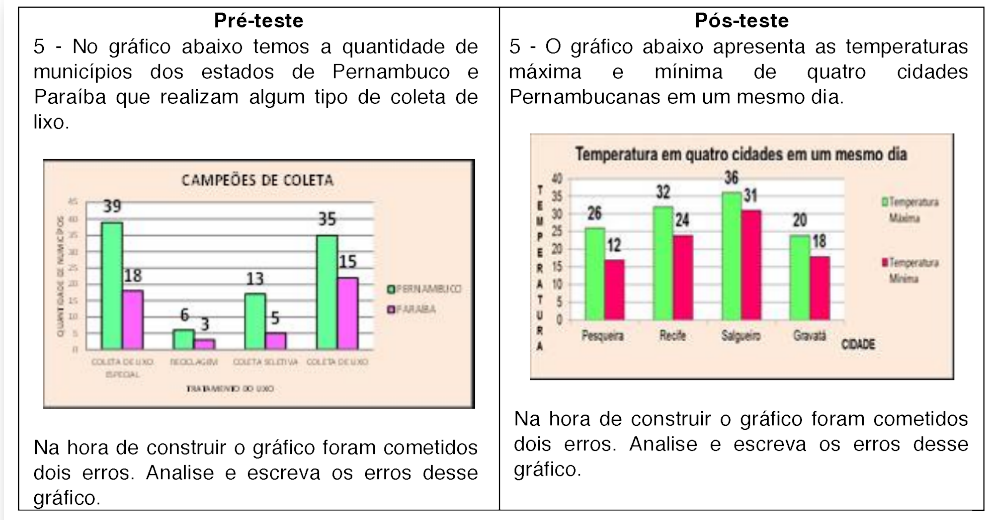

Figura 8 - Quinta questão do pré-teste e do pós-teste

Acreditamos que a dificuldade dos alunos no pré-teste em localizar os erros pode ter sido também em decorrência da ausência de familiaridade dos mesmos com esse tipo de atividade. Cavalcanti et al. (2010) afirmam que os gráficos vinculados pela mídia impressa priorizam colocar os valores em cima das barras, o que dispensa os leitores de compreenderem a escala. Entretanto, esse tipo de veiculação pode mais facilmente disfarçar a ausência de proporção da escala do gráfico, permitindo uma ideia equivocada das informações apresentadas. Por isso, Friel, Curcio, e Brigh (2001) destacam a 
necessidade de reconhecer os elementos que estruturam um gráfico (eixo, escala, etiquetas e outros elementos específicos) e a inter-relação entre esses componentes e os seus efeitos na apresentação da informação em gráfico.

$\mathrm{Na}$ sexta questão foi solicitado aos alunos que realizassem a correspondência entre valores expressos em um gráfico e em uma tabela (Figura 9).

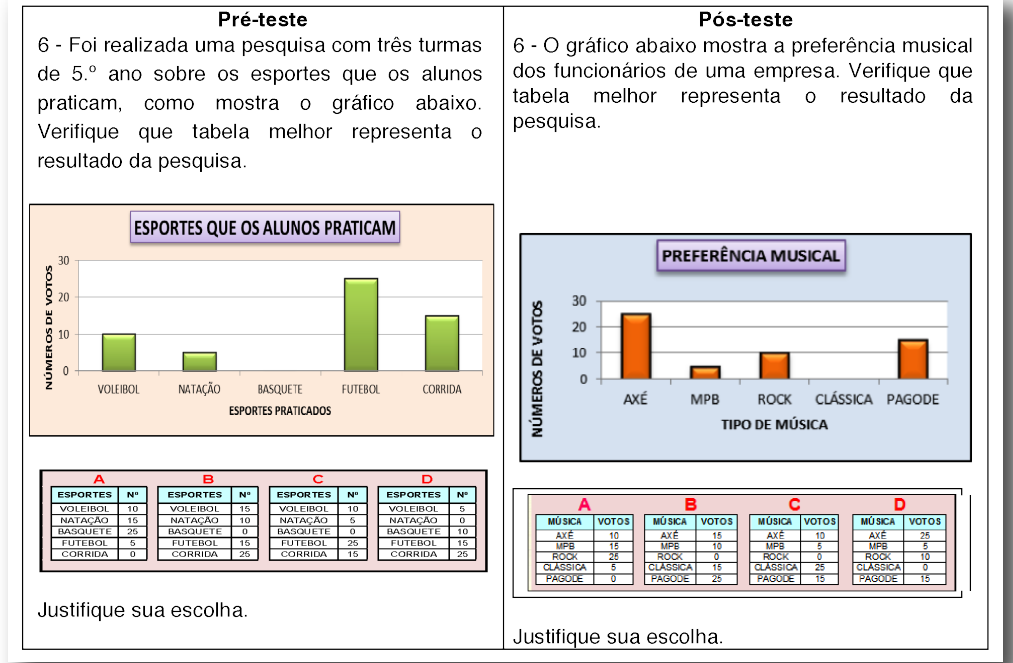

Figura 9 - Sexta questão do pré-teste e do pós-teste

Esse tipo de atividade é encontrado com frequência em livros didáticos, o que pode explicar o bom desempenho de todos os grupos de alunos já no pré-teste (MC 50\%; RM 65,2\%; MP 54,4\%), com alterações mais discretas no pós-teste (MC 46\%; RM 78\%; MP 64\%). Esses resultados reforçam os apresentados por Magina, Cazorla, e Pagan (2009), os quais evidenciam que $50 \%$ dos alunos do $5 .^{\circ}$ ano conseguiram realizar conversões de gráficos para tabelas. Silva (2012) também reforça esses resultados; porém, ressalta que alunos com menor idade/escolaridade, ou seja, alunos do 3..$^{\circ}$ ano (8 anos de idade) apresentaram mais dificuldade, uma vez que 
apenas 37\% conseguiram acertar. Para nós, esses dados evidenciam que alunos no início de escolarização já são capazes de compreender essa relação, cabendo aos professores trabalhar de forma mais sistemática para uma maior e melhor apropriação de todos.

$\mathrm{Na}$ sétima questão (Figura 10), foi solicitado aos alunos que comparassem gráficos que apresentavam os mesmos dados, mas com escalas diferentes, e que justificassem suas respostas.

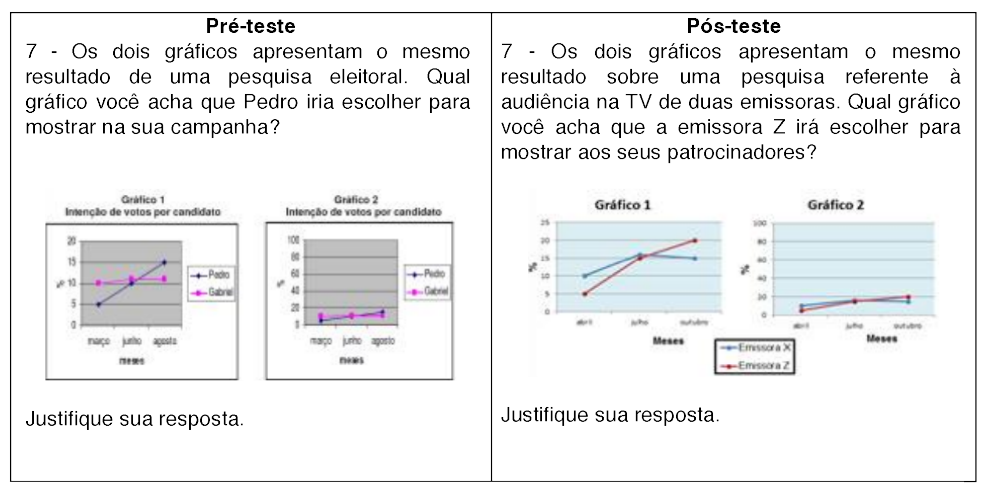

Figura 10 - Sétima questão do pré-teste e do pós-teste

Esta atividade foi muito difícil para os alunos, tanto no pré como no pós-teste. Apenas um aluno no pré e 4 no pós-teste foram capazes de perceber a influência da escala. Essa atividade foi proposta por Albuquerque (2010), a qual também percebeu, em seu estudo, que tanto crianças como adultos que frequentavam os anos iniciais de escolarização apresentavam muitas dificuldades. Acreditamos que apesar dessa dificuldade, esse tipo de atividade precisa ser proposto aos alunos para que os mesmos reflitam sobre essa variação. Como afirmam Cavalcanti et al. (2010), é fundamental ter um olhar mais crítico sobre as informações que são veiculadas em nosso dia a dia, pois frequentemente aparecem na mídia informações em gráficos com escala manipuladas, as quais podem enfatizar, mascarar ou omitir determinados aspectos da notícia em função da intenção de quem a estruturou. 
$\mathrm{Na}$ oitava questão dos dois testes, foi solicitado aos alunos que construíssem um gráfico de barras a partir de uma tabela (Figura 11).

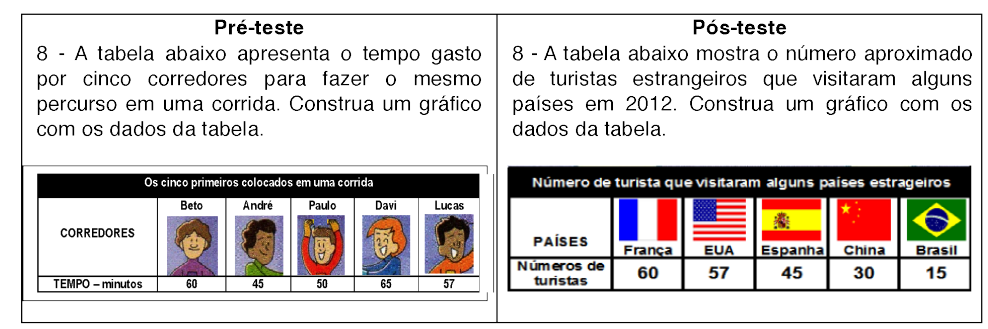

Figura 11 - Oitava questão do pré-teste e do pós-teste

Verificamos no pré-teste que os grupos não apresentaram um bom desempenho (MC 8\%; RN 17\%; MP 9\%). No entanto, após a intervenção de ensino, constatamos avanços para os grupos MC (38\%) e MP (18\%). Já o RN não conseguiu melhorar seu desempenho, apresentando rendimento igual ao do teste inicial.

Como afirmam Silva e Guimarães (2013), a interpretação de escalas é uma habilidade tão importante quanto a de construir escalas e a compreensão de uma ajuda na compreensão da outra. Dessa forma, é necessário um maior destaque na proposição de atividades de construção de escalas.

Nossos resultados corroboram estudos anteriores que afirmam que os alunos sentem bastante dificuldade para construir gráficos com escalas proporcionalmente adequadas, como observado por Guimarães (2002) com alunos do $4 .^{\circ}$ ano, Silva (2012) com alunos do $3 .^{\circ}$ e $5 .^{\circ}$ anos de escolarização e Lima (2010) com alunos da Educação de Jovens e Adultos.

Ressaltamos, ainda, que as situações apresentadas na intervenção não levaram a tipos de aprendizagem diferentes. Assim, a capacidade de adaptação dos alunos em estudar escala em atividades de um eixo matemático e transferi-la para outro é evidenciada, uma vez que as situações de intervenção envolviam contextos de medidas, mapas e reta numérica e os testes envolviam representações em gráficos de barra e linha. 


\section{Conclusões}

Nesse estudo investigámos a possibilidade de aprendizagem sobre escala representada em gráficos de barras e linhas, por alunos do $5 .^{\circ}$ ano do Ensino Fundamental, a partir de três tipos de situação: medida de comprimento, reta numérica e mapas.

Os alunos, inicialmente, apresentaram um fraco desempenho, demonstrando dificuldades, como já havia sido observado em outros estudos (Guimarães, 2002; Lemos, 2002; Albuquerque, 2010; Lima, 2010; Silva, 2012; entre outros). Entretanto, após terem sido submetidos à intervenção de ensino, percebemos que houve um avanço significativo no seu desempenho sobre escalas representadas em gráficos de barras e de linha simples, independentemente do tipo de contexto (medida de comprimento, reta numérica e mapas) explorado na intervenção.

Tais resultados nos parecem muito importantes, uma vez que expressam a facilidade que as crianças apresentam em aprender sobre escalas quando são estimuladas de forma sistemática. Além disso, podemos afirmar que os três tipos de contexto por nós propostos (medida de comprimento, reta numérica e mapas), os quais envolvem diferentes eixos da matemática, permitiram a aprendizagem sobre escalas e a compreensão dessas representadas em gráficos de linha e barra. Dessa forma, parece que uma intervenção sobre escalas pode minimizar diferenças encontradas em estudos anteriores (Albuquerque, 2010; Bezerra \& Guimarães, 2013), entre gráficos de barras e linhas. Concluímos, assim, que este estudo contribui de forma clara para demonstrar a necessidade e a possibilidade de um trabalho sistemático na escola, desde os anos iniciais, relativo à apropriação de escalas.

\section{Notas}

1 Programa Nacional do Livro Didático - Esse Programa do Ministério de Educação do Brasil tem por objetivo prover as escolas públicas de Ensino Fundamental e Médio com livros didáticos e acervos de obras literárias, obras complementares e dicionários. Um edital especifica todos os critérios para inscrição das obras. Os títulos inscritos pelas editoras são avaliados pelo MEC, que elabora o Guia do Livro Didático, composto das resenhas de cada obra aprovada e disponibilizado às escolas participantes pelo Fundo Nacional de Desenvolvimento da Educação (FNDE).

2 A representação refere-se a um segmento de reta, apesar de ser nomeada como reta. 


\section{Referências}

Ainley, J. (2000). Exploring the transparency of graphs and graphing. In T. Nakahara \& M. Koyama (Eds.), Proceedings of the 24th Annual Conference of the International Group for the Psychology of Mathematics Education, (vol. 2, pp. 916). Hiroshima, Japão.

Albuquerque, R. G. C. (2010). Como adultos e crianças compreendem a escala representada em gráficos (Dissertação de mestrado). Universidade Federal de Pernambuco, Recife - PE.

Bezerra, L., \& Guimarães, G. (2013). Compreensão de escalas representadas em gráficos por alunos adultos pouco escolarizados. In J. M. Contreras, G. R. Cañadas, M. M. Gea, \& P. Arteaga (Eds.), Actas de las Jornadas Virtuales em Didáctica de la Estadística, Probabilidad y Combinatoria (pp. 143-148). Granada: Departamento de Didáctica de la Matemática de la Universidad de Granada.

Boaventura, M. G., \& Fernandes, J. A, (2004). Dificuldades de alunos do $12 .^{\circ}$ ano nas medidas de tendência central: $O$ contributo dos manuais escolares. In J. A. Fernandes, M. V. Sousa, \& S. A. Ribeiro (Orgs.), Actas do I Encontro de Probabilidades e Estatística na Escola (pp. 103-126). Braga: CIEd, Universidade do Minho.

Bruno, A., \& Espinel, M. C. (2005). Recta numérica, escalas y gráficas estadísticas: Un estudio con estudiantes para profesores. Formación del Profesorado e Investigación en Educación Matemática VII 57-85.

Cavalcanti, M. R. G., Natrielli, K. R. B., \& Guimarães, G. L. (2010). Gráficos na mídia impressa. Bolema, 23(36), 733-751.

D’Ambrósio, U. (2007). A relevância do projeto 'Indicador Nacional de Alfabetismo Funcional - INAF' como critério de avaliação da qualidade do ensino de matemática. In M. C. Fonseca (Org), Letramento no Brasil: Habilidades matemáticas (pp. 31-46). São Paulo: Global Editora.

Evangelista, M. B., \& Guimarães, G. L. (2013). Análise de atividade de livros didáticos de matemática do $4^{\circ}$ e $5^{\circ}$ ano que exploram o conceito de escala. In Anais do VII Congreso Iberomericano de Educación Matemática - CIBEM. Montevideo, Paraguai.

Friel, S., Curcio, F., \& Bright, G. (2001). Making sense of graphs: Critical factors influencing comprehension and instructional implications. Journal for Research in Mathematics Education, 32(2), 124-158.

Guimarães, G. L. (2002). Interpretando e construindo gráficos de barras (Tese de Doutorado). Universidade Federal de Pernambuco, Recife - PE.

Guimarães, G. L., Ferreira, V. G. G., \& Roazzi, A. (2001). Interpretando e construindo gráficos. In Anais da $24^{a}$ Reunião Anual da ANPED - GT Educação Matemática. Caxambu - MG. http://www.ufrrj.br/emanped/paginas/conteudo/producoes/ docs_24/ interpretando.pdf (acesso em 16 de abril de 2012).

Guimarães, G. L., Gitirana, V. G. F., Cavalcanti, M., \& Marques, M. (2007). Livros didáticos de matemática nos anos iniciais: Análise das atividades sobre gráficos e tabelas. In Anais do IX Encontro Nacional de Educação Matemática. Belo Horizonte, MG. 
Lemos, M. P. (2002). Professorandos analisando atividades de interpretação de gráficos de barras (Dissertação de mestrado). Universidade Federal de Pernambuco, Recife, PE.

Lima, I. B. (2010). Investigando o desempenho de jovens e adultos na construção e interpretação de gráficos (Dissertação de mestrado). Universidade Federal de Pernambuco, Recife, PE.

Lima, R. C. R., \& Magina, S. M. (2010). O uso de diferentes escalas na leitura de gráficos por crianças das séries iniciais do Ensino Fundamental. In Anais do IX Encontro Nacional de Educação Matemática - X ENEM. Salvador - BA. http://www.sbem. com.br/ocs/index.php/xenem/xenem/paper/view/1707 (acesso em 5 de setembro de 2012).

Magina, S., Cazorla, I., \& Pagan, A. (2009). Conversão de registros na construção de tabelas e gráficos: Estudo comparativo entre alunos do ensino básico. In Anais do VI Congreso Iberoamericano de Educación Matemática (vol. 1, pp. 475-476). Puerto Montt: Sociedad Chilena de Educación Matemática.

Melo, M. S. L., \& Bellemain, P. M. B. (2006). A abordagem do conceito de escala em livros didáticos para o terceiro e quarto ciclos do Ensino Fundamental: Uma análise à luz da teoria dos campos conceituais. In Anais do Simpósio Internacional de Pesquisa em Educação Matemática - SIPEMAT. Recife - PE: Sociedade Brasileira de Educação Matemática. Disponível em http://www.lematec.net/CDS/SIPEMAT06/artigos/melobellemain.pdf.

Melo, M. S. L., \& Bellemain, P. M. B. (2009). O ensino e a aprendizagem do conceito de escala, no quarto ciclo do Ensino Fundamental, à luz da teoria dos campos conceituais. In Anais do VIII Encontro Nacional de Educação Matemática ENEM. Recife - PE: Sociedade Brasileira de Educação Matemática. Disponível em http://www.sbembrasil.org.br/files/viii/pdf/02/PO47377429453 .pdf.

Ministério da Educação e do Desporto/Secretaria de Educação Fundamental (1997). Parâmetros Curriculares Nacionais de Matemática do Ensino Fundamental I$1^{\mathrm{a}}$ à $4^{\mathrm{a}}$ série. Brasília: MEC/SEF.

Monteiro, C. (2006). Estudantes de Pedagogia refletindo sobre gráficos da mídia impressa. In Anais do I Seminário Internacional de Pesquisa em Educação Matemática - I SIPEMAT. Recife, PE.

Ponte, J. P., Brocardo, J., \& Oliveira, H. (2009). Investigações matemáticas na sala de aula ( $2^{\mathrm{a}}$ ed.). Belo Horizonte: Autêntica.

Silva, D. B. (2012). Analisando a transformação entre gráficos e tabelas por alunos do $3^{\circ}$ e $5^{\circ}$ ano do Ensino Fundamental (Dissertação de mestrado). Universidade Federal de Pernambuco, Recife - PE.

Silva, E. M. C., \& Guimarães, G. L. (2013). Livros didáticos para os anos iniciais do Ensino Fundamental propõem aos alunos que realizem pesquisas?. In Anais do XV EBRAPEM - Encontro Brasileiro de Estudantes de Pós-Graduação em Educação Matemática. Campina Grande, PB. 


\title{
SCALES REPRESENTED IN GRAPHS: AN INTERVENTION STUDY WITH
} STUDENTS OF THE 5TH GRADE

\begin{abstract}
This article presents a study that investigated learning about scales represented in bar and line graphs after a teaching intervention. 69 students of the 5 th grade (about 10 years old) of three Brazilian public schools participated in this study. For each group, activities involving different situations were proposed: length measure, number line and maps. All students participated in a pre-test, a teaching intervention and a post-test. The results revealed that students have difficulties to represent, locate, analyze, compare and build scales on graphs. However, after only two intervention sessions in each group, significant improvements were observed in the learning of all groups. Thus, we can say that students in primary school, when involved in thinking about scales, are able to learn about that representation. In that way, the need and possibility of a systematic work in schools is evidenced.
\end{abstract}

Keywords

Scale; Graph; Primary School; Mathematics

\section{ÉCHELLES REPRÉSENTÉES DANS LES GRAPHIQUES : UNE ÉTUDE D'INTERVENTION AVEC DES ÉLÈVES DU 5EME ANNÉE}

\author{
Résumé \\ Cet article présente une étude qui s'est intéressée à l'apprentissage de \\ l'échelle chez les enfants lorsqu'elle est représentée dans les graphiques à \\ barres et des lignes. Ont participé de cette étude 69 élèves (environ 10 ans) \\ de trois écoles publiques au Brésil. Dans chacun des groupes, il a été proposé \\ des activités impliquant différentes situations: mesure de longueur, droite \\ numérique et des cartes. Tous les élèves ont participé à un pré-test, deux
}


séances d'enseignement et un post-test. Les résultats du pré-test montrent que les élèves présentent des difficultés à représenter, localiser, analyser, comparer et construire l'échelle dans le graphique. Néanmoins, seulement après deux séances d'intervention dans chaque groupe, il a été observé une progression significative concernant de l'apprentissage d'échelles chez ces élèves dans tous les groupes. Ainsi, il est possible d'affirmer que lorsque les élèves de l'école primaire sont amenés à réfléchir sur les échelles, ils sont en mesure d'apprendre sur l'échelle aux graphiques à barres et des lignes.

Mots-clé

Échelle; Graphique; École primaire; Mathématiques

Recebido em julho/2014

Aceite para publicação em maio/2015

i Grupo de Estudo em Educação Estatística no Ensino Fundamental - GREF, Universidade Federal de Pernambuco, Brasil.

ii Centro de Educação, Departamento de Métodos e Técnica de Ensino, Universidade Federal de Pernambuco, Brasil.

Toda a correspondência relativa a este artigo deve ser enviada para: Maria Betânia Evangelista, Rua Cônego Barata, 908 - BI A5 apto 403, CEP 52110-120 - Recife/PE, Brasil. E-mail: mbevangelista@hotmail.com 